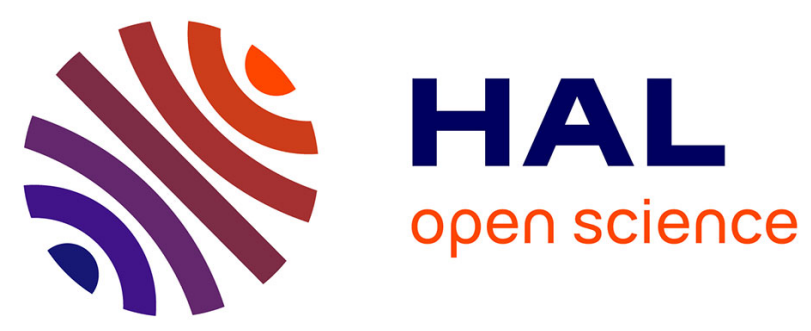

\title{
Nonlinear optical spectra of confined and localized excitons in mesoscopic domain and quasi-2D stacking fault interface in BiI3 crystals
}

\author{
I. Akai, H. Kondo, M. Ichida, T. Karasawa, T. Komatsu
}

\section{To cite this version:}

I. Akai, H. Kondo, M. Ichida, T. Karasawa, T. Komatsu. Nonlinear optical spectra of confined and localized excitons in mesoscopic domain and quasi-2D stacking fault interface in BiI3 crystals. Journal de Physique IV Proceedings, 1993, 03 (C5), pp.C5-433-C5-436. 10.1051/jp4:1993593 . jpa-00251680

\author{
HAL Id: jpa-00251680 \\ https://hal.science/jpa-00251680
}

Submitted on 1 Jan 1993

HAL is a multi-disciplinary open access archive for the deposit and dissemination of scientific research documents, whether they are published or not. The documents may come from teaching and research institutions in France or abroad, or from public or private research centers.
L'archive ouverte pluridisciplinaire HAL, est destinée au dépôt et à la diffusion de documents scientifiques de niveau recherche, publiés ou non, émanant des établissements d'enseignement et de recherche français ou étrangers, des laboratoires publics ou privés. 


\title{
Nonlinear optical spectra of confined and localized excitons in mesoscopic domain and quasi-2D stacking fault interface in $\mathrm{BiI}_{3}$ crystals
}

\author{
I. AKAI, H. KONDO, M. ICHIDA, T. KARASAWA and T. KOMATSU
}

Department of Physics, Faculty of Science, Osaka City University, 3-3-138 Sugimoto Sumiyoshi-ku Osaka 558, Japan

\begin{abstract}
Saturation spectra of the excitons confined and localized at several kinds of stacking disorders in layered crystal $\mathrm{BiI}_{3}$ are studied. The quasi-two dimensional exciton localized at the stacking fault interface does not saturate its absorption strength below the density of $10^{5} \mathrm{~W} / \mathrm{cm}^{2}$ due to the large binding energy and the lateral center-of-mass motion along the interface. On the exciton system associated with some polytypic structures contained in a very small region, an obvious absorption saturation is observed. The behaviour of absorption saturation is well described by a saturation effect on a simplified two-level system having a homogeneous line broadening. An absorption saturation is also observed on the confined excitons in mesoscopic domains induced by the external deformation of the crystal. The pumping-laser photon-energy dependence of the saturation density characterizes this exciton system to be inhomogeneous.
\end{abstract}

\section{INTRODUCTION}

Spatially restrictions of the relative motion and the center-of-mass motion of excitons make us possible to control the optical response of the excitons. We can expect the enhancement of the nonlinear optical effect and the exhibition of new nonlinear phenomena in the spatially restricted exciton systems.

In layered crystal $\mathrm{BiI}_{3}$, sharp absorption peaks due to several kinds of spatially restricted exciton have been observed. Figure 1 shows the typical absorption spectrum of $\mathrm{BiI}_{3}$. A stacking fault, which keeps the translational symmetry along the stacking fault plane, gives rise to a set of sharp absorption lines named $R, S$, and $T{ }^{[1]}$ They have been called stacking fault excitons (SFE's). ${ }^{[1]}$ The coherent non-

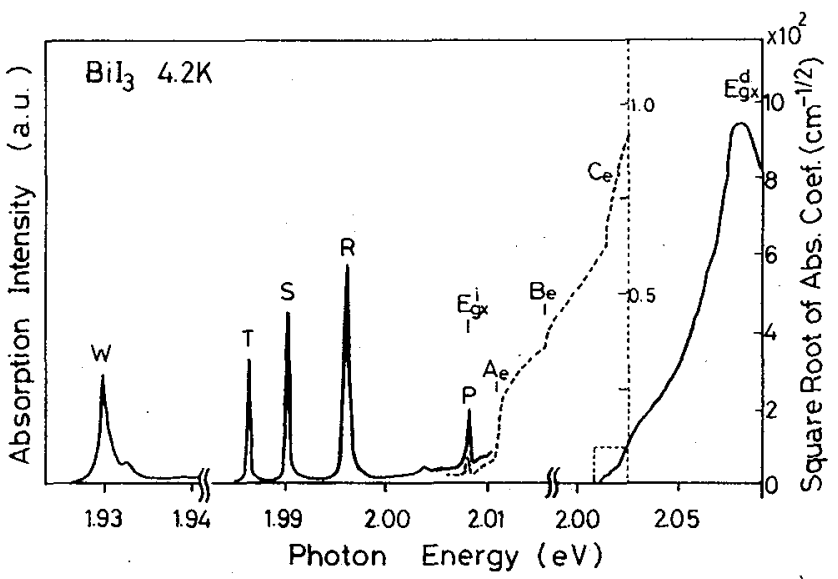

Figure 1: Typical absorption spectrum of $\mathrm{BiI}_{3}$ crystal at $4.2 \mathrm{~K} . E_{g x}^{d}$ and $E_{g x}^{i}$ : the transition energy of the direct and indirect exciton, respectively. $A_{e}, B_{e}$, and $C_{e}$ :the absorption step of the indirect exciton assisted with phonon $A, B$, and $C$ emission, respectively. $P, R, S, T$, and $W$ : see text. 
linear interaction of these excitons with photon field was reported in terms of optical Stark effects on the exciton system in previous papers. ${ }^{[2]}$ The nonlinear effect due to the interaction among the really excited excitons was also reported. ${ }^{[3]}$ The external stress and/or bending on the crystal bring about broad absorption bands called $W$-band in the energy region lower than the SFE transitions. ${ }^{[1]}$ The $W$-band has been ascribed to the transitions of excitons confined in mesoscopic domain structures induced by the external deformation in the crystals. The spectral profile of the $W$-band is caused by the size distribution of the mesoscopic domains. Near the indirect exciton transition energy, a sharp absorption line named $P$ also appears depending on samples. The origin of the $P$-line has been assigned to the exciton associated with some polytypic structures contained in a very small region. [4]

In this paper, a saturation spectroscopy under the intense laser excitation on these exciton systems in $\mathrm{BiI}_{3}$ crystal is performed. We will discuss the relation between the behaviour of the saturation effect and the spatially restricted effect on these excitons.

\section{EXPERIMENTAL}

High quality crystals of $\mathrm{BiI}_{3}$ were selected from the absorption measurements of these exciton transitions (SFE, $P$, and $W$ ). All measurements were performed at $4.2 \mathrm{~K}$ and $2 \mathrm{~K}$. A dye laser (Rhodamine $\mathrm{B}$ and Rhodamine 640) pumped by a nitrogen laser was used. For measuring the excitation density dependence of the absorption spectra, we change the laser intensity in the range of $10^{2} \sim 10^{6} \mathrm{~W} / \mathrm{cm}^{2}$.

\section{RESULTS AND DISCUSSION}

The absorption for the pumping laser light on the SFE, the $P$-exciton transition, and the $W$-band change depending on the excitation density $I$ of the pumping laser. Line broadenings and peak-energy shifts to higher energy side are observed on the absorption spectra of these exciton states obtained by scanning the pumping laser photon energy.

Figure 2 shows the excitation density dependences of the absorption strength $f(I)\left({ }^{1}\right)$ of the transitions of $R$ and the $P$-line. The absorption strength of the $R$-line does not change below the excitation density of $1 \times$ $10^{5} \mathrm{~W} / \mathrm{cm}^{2}$. On the large Bohr-radius

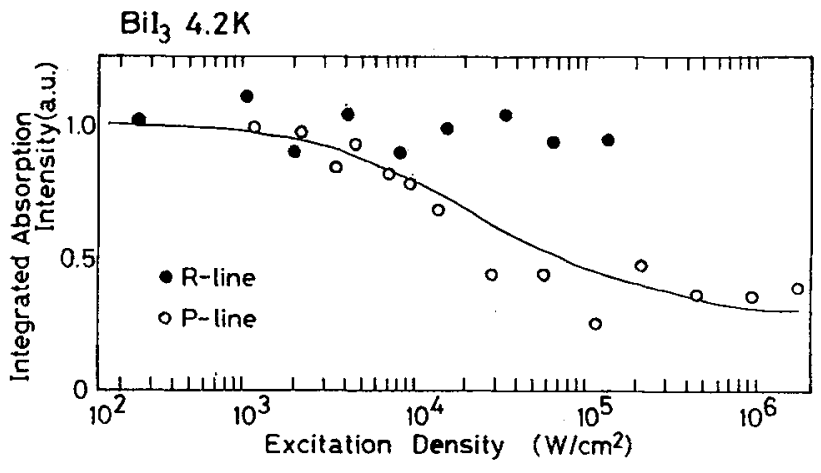

Figure 2: Excitation density dependences of the absorption strength of $R$-line and $P$-line. Ordinate denotes the normalized absorption strength by the strength under the weak excitation limit. excitons in GaAs materials, the saturations of the exciton absorption occur below the excitation density of $\sim 1 \times 10^{4} \mathrm{~W} / \mathrm{cm}^{2}$. ${ }^{[5]}$ The excitons in $\mathrm{BiI}_{3}$ have large binding energy and small exciton Bohr radius contrary to the exciton in $\mathrm{GaAs}$ reflecting the cationic nature ${ }^{[1]}$ of bulk exciton. This nature suggests that it needs higher intensity of laser light to realize the exciton saturation contrary to the case in GaAs.

On the contrary, the $P$-line saturates its absorption strength above the excitation density of $2 \times 10^{3} \mathrm{~W} / \mathrm{cm}^{2}$ and fades out above the density of $\sim 10^{5} \mathrm{~W} / \mathrm{cm}^{2}$. For higher density excitation, a finite absorption remains. This component is due to a nonsaturable background. The smallness of saturation density in the $P$-lines is considered to be due to the spatially restricted effect of the $P$-exciton. The SFE is considered to be exempted from the exciton saturation because of the degree of freedom due to the lateral center-of-motion along the stacking fault interface. This consideration

$\left(^{1}\right) f(I)=\int \alpha(\hbar \omega, I) d \hbar \omega$, where $\hbar \omega$ denotes the pumping laser photon energy, $I$ : the excitation density 
implies that the $P$-exciton state is deprived of the degree of freedom and localized at very small region in crystal. The facts are consistent with the result of the magnet-optical measurement. [4]

Neglecting the small peak shift, for simplify, we obtain the saturation density $I_{S}(\hbar \omega)$ as a function of the pumping laser photon energy $\hbar \omega$. The saturation density $I_{S}(\hbar \omega)$ is defined as follows:

$$
\alpha\left(I_{S}(\hbar \omega), \hbar \omega\right) \equiv \frac{1}{2}\left[\alpha(0, \hbar \omega)+\alpha_{B}(\hbar \omega)\right],
$$

where $\alpha(I, \hbar \omega)$ is the absorption coefficient for the pumping laser, $\alpha_{B}(\hbar \omega)$ is the absorption intensity due to a nonsaturable background. Figure 3 shows the $\hbar \omega$ dependence of $I_{S}$ of the $P$-line. The saturation density $I_{S}(\hbar \omega)$ near the $P$-resonance has the minimum value of $1.1 \times 10^{4} \mathrm{~W} / \mathrm{cm}^{2}$ and increases with detuning laser frequency.

Assuming the $P$-exciton state to be a simple twolevel system, we can analyze the observed $\hbar \omega$ dependence of $I_{S}$ as a saturation effect accompanying power broadening. The absorption coefficient $\alpha(I, \hbar \omega)$ for the pumping laser is given as a following form: ${ }^{[6]}$

$$
\alpha(I, \hbar \omega)=\frac{\alpha_{0} \Gamma^{2}}{(\omega-\Omega)^{2}+\Gamma^{2}\left(1+I / I_{S}^{0}\right)},
$$

where $\alpha_{0}$ is the peak absorption coefficient without

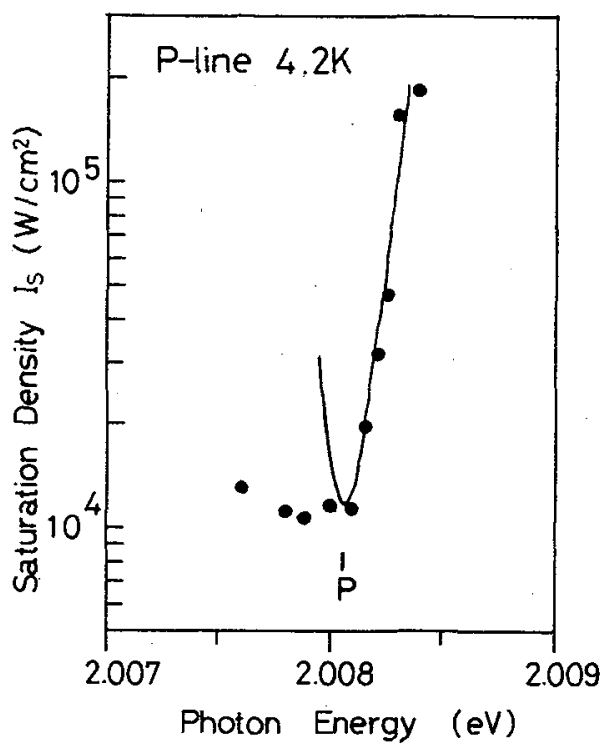

Figure 3: $\hbar \omega$ dependences of the saturation density $I_{S}(\hbar \omega)$ on the $P$-line. The solid line is theoretical fit (see text).

pumping, $\Omega$ : resonant frequency of the exciton concerned, $\Gamma$ : damping constant giving the homogeneous line width, $I_{S}^{0}$ : the saturation density under the just resonant condition $(\omega=\Omega$ in Eq.(1)). One obtains the $\hbar \omega$ dependence of $I_{S}(\hbar \omega)$ as:

$$
I_{S}(\hbar \omega)=\left(1+\frac{(\omega-\Omega)^{2}}{\Gamma^{2}}\right) I_{S}^{0}
$$

The solid line in Fig. 3 is the plot of $I_{S}(\hbar \omega)$ in Eq.(3) from the $I_{S}^{0}$ of $1.1 \times 10^{4} \mathrm{~W} / \mathrm{cm}^{2}$, where we take the values of $\Omega$ and $\Gamma$ to the values without pumping neglecting the peak shift and lifetime broadening due to the interaction among the really excited excitons. The saturation density obtained from Eq.(3) takes the minimum value at $\omega=\Omega$ and increases with increasing the detuning frequency. This plot well reproduces the experimental results. Thus we can evaluate the excitation-density dependence of the absorption strength $f$ plotted in Fig.2. From Eq.(2), we obtain $f(I)$ using the $I_{S}^{0}$ as follows:

$$
f(I)=\frac{f_{0}}{\sqrt{1+I / I_{S}^{0}}} .
$$

The calculated $f(I)$ is shown in Fig.2 by using the above value for $I_{S}^{0}$, where a nonsaturable background is taken into account. The calculation well reproduces the experimental plots.

An absorption saturation is also observed in the $W$-band. Figure 4 shows the $\hbar \omega$ dependence of the saturation density $I_{S}$ of the $W$-band. Contrary to the $P$-line, the value of $I_{S}(\hbar \omega)$ increases monotonously with increasing $\hbar \omega$. As mentioned in introduction, the $W$-band has been ascribed to the excitons confined in mesoscopic domains of various size in the crystal. The size-distribution gives the inhomogeneous width.

The absorption coefficient $\alpha(I, \hbar \omega)$ for the pumping laser is given as a following form in the case of an explicit inhomogeneous system: ${ }^{[6]}$ 
$\alpha(I, \hbar \omega)=h(\omega, I) \int g(\omega, \Omega, I) d \Omega=\frac{h(\omega, I)}{\sqrt{1+I / I_{S}^{0}(\omega)}}$,

where $h$ is an inhomogeneous broadening function, $g(\omega, \Omega, I)$ : a normalized homogeneous broadening function having the resonance at $\Omega, I_{S}^{0}(\omega)$ : the resonance saturation density of the state having its resonance at $\omega$. In an inhomogeneous system, the behaviour of the saturation effect is governed by both $h(\omega, I)$ and $I_{S}^{0}(\omega)$. Energy immigration and relaxation among homogeneous states induce the deformation of the function $h$ for the intense laser excitation. In this experiment, since we use the nanosecond light pulse of relatively long time duration, the energy immigration and relaxation occur. This process has been observed on the time resolved measurements of the $W$-band luminescence in the picosecond time region. This fact means that the lower lying states are easily saturated. Then, it is considered that this relaxation considerably contributes to the monotonous decrease of $I_{S}(\hbar \omega)$ for decreasing $\hbar \omega$.

The difference of the confined effect on the excitons in the mesoscopic domains with different sizes gives the different value of $I_{S}^{0}$ for different $\omega$. In the

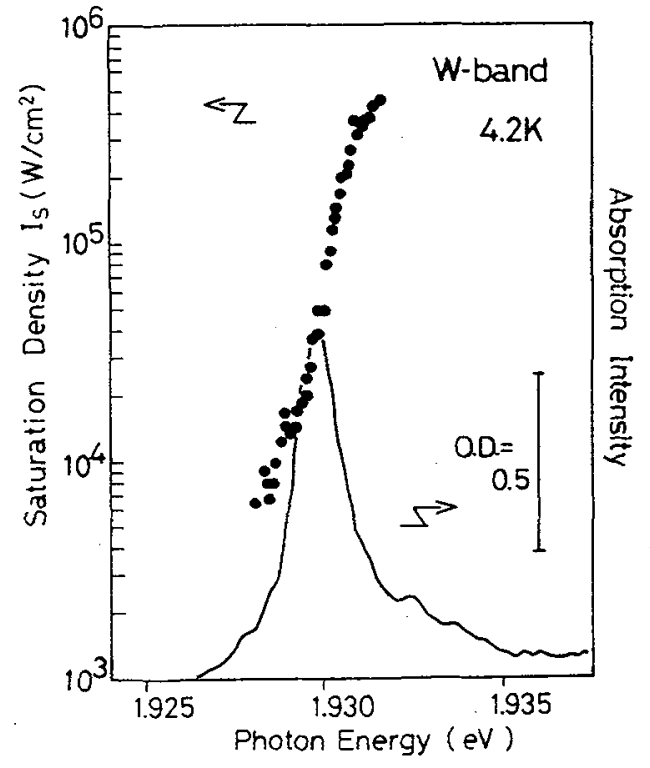

Figure 4: Pumping laser photon energy dependences of the saturation density $I_{S}$ on the W-band. The absorption spectrum under the weak excitation limits is drawn for eye-guide.

W-band, the lower lying states comes from the exciton confined in larger mesoscopic domains. The experimental plots in Fig.4 show that the saturation density decreases with increasing the mesoscopic domain size. Knowing the the domain size dependence of the absorption cross-section and the size distribution of the mesoscopic domains, we will make clear the confined effect on the nonlinear optical response of the $W$-excitons.

\section{SUMMARY}

A saturation-spectroscopy study on the excitons confined and localized at several kinds of stacking disorders in layered crystal $\mathrm{BiI}_{3}$ is made. The SFE excitons do not saturate their absorption strengths due to their large binding energy and the lateral center-of-motion along the stacking fault interface. The behaviour of absorption saturation in the $P$-line is well described by a saturation effect on a simplified two-level system having a homogeneous line broadening. The $\hbar \omega$ dependence of the $I_{S}$ in the $W$-band is considered to be due to the energy relaxation among the inhomogeneouslly broadened exciton states and due to the mesoscopic domain size dependence of the saturation density.

\section{References}

[1] Kaifu Y., J. Lumin. 42 (1988) p. 61.

[2] Akai I., Karasawa T. and Komatsu T., Int. J. Nonlinear Opt. Phys. 1 (1992) p. 311.

[3] Karasawa T., Ichida M., Akai I. and Komatsu T., Appl. Phys. A53 (1991) p. 480.

[4] Komatsu T., Koike K., Kaifu Y., Takeyama S., Watanabe K. and Miura N., Phys. Rev. B48 (1993) now printing.

[5] Peyghambarian N. and Koch S. W., Nonlinear Photonics (30 of Springer Series in Electronics and Photonics Springer-Verlag 1990) p. 26.

[6] Akulin V. M. and Karlov N. V., Intense Resonant Interaction in Quantum Electronics SpringerVerlag Berlin Heidelberg (1992) pp. 43-65. 